\title{
WEIERSTRASS PREPARATION THEOREM
}

\author{
H. SERBIN
}

H. Späth ${ }^{1}$ has shown that the Weierstrass preparation theorem is a consequence of the following one:

THEOREM. Let $P$ and $Q$ be power series in the variables $z_{1}, z_{2}, \cdots, z_{m}$ such that $P$ is regular in $z_{1}$ of degree $k$, that is, $P\left(z_{1}, 0, \cdots, 0\right)$ $=c z_{1}^{k}+d z_{1}{ }^{k+1}+\cdots,(c \neq 0)$. Then there exist power series $A$ and $B$ such that

$$
Q-A P=B
$$

where $B$ does not contain powers of $z_{1}$ higher than the $(k-1)$ st. The series $A$ and $B$ are uniquely determined.

It has not been observed that this general form permits of a simple proof by induction when we are concerned only with formal series. The solution of (1) is equivalent to the solution of the system

$$
\begin{aligned}
\left(q_{n}-a_{0} p_{n}-a_{1} p_{n-1}-\cdots-a_{n-1} p_{1}\right)-a_{n} p_{0} & =b_{n}, \\
n & =0,1,2, \cdots,
\end{aligned}
$$

where $P=\sum p_{n} z_{m}{ }^{n}, Q=\sum q_{n} z_{m}{ }^{n}, A=\sum a_{n} z_{m}{ }^{n}, B=\sum b_{n} z_{m}{ }^{n}$. The theorem is evidently true for the case $m=1$. Since $p_{0}$ is regular in $z_{1}$ of degree $k$, the existence of a formal solution of (1) and its uniqueness is an immediate consequence of an induction from $m-1$ to $m$ variables.

Princeton, N.J.

${ }^{1} \mathrm{H}$. Späth, Journal für die reine und angewandte Mathematik, vol. 161 (1929), pp. 95-100. This contains additional references. 